\title{
Iberia-Azores Gravity Model (IAGRM) using multi-source gravity data
}

\author{
J. Catalao \\ LATTEX, University of Lisbon, Faculty of Sciences, Campo Grande, 1749-016 Lisbon, Portugal
}

(Received April 20, 2005; Revised September 15, 2005; Accepted September 27, 2005; Online published March 10, 2006)

\begin{abstract}
A consistent high precision and high resolution gravity model in the north-east Atlantic off Iberia peninsula using multi-source gravity data, ship-borne and satellite derived gravity anomalies, is presented. A solution strategy based on least squares optimal interpolation was used to assimilate into a coherent gravity model, gravity data with different spectral and spatial resolutions. Satellite derived gravity anomalies from KMS02 model, with an error covariance of $25 \mathrm{mGal}^{2}$, and marine data carefully edited and validated by bias crossover error adjustment, were used in this study. The observation error variance was determined from ship-borne track adjustment and assigned an independent value for each track determined from error variance propagation. Unbiased ship-borne gravity observations were assimilated into the satellite derived gravity KMS02 model by the least squares optimal interpolation algorithm (OI) with bias removed by applying a regional bias to all ship tracks (OI-b) and alternatively by constraining all ship tracks to KMS02 using bias and tilt (OI-t). External error of the model was determined by comparing with recent surveys and it was verified that OI-t approach improved the final gravity model to an accuracy of about $3 \mathrm{mGal}$. The effect of different merging approaches on geoid solution was also evaluated and it was verified that the merging process can contribute to improve the geoid accuracy up to 4 $\mathrm{cm}$ with the OI-t approach.
\end{abstract}

Key words: Gravity, merging techniques, Iberia-Azores, optimal interpolation.

\section{Introduction}

New methods and tools to support gravity data collection were developed worldwide in the last ten years (Childers et al., 2001; Forsberg and Brozena, 1992; Forsberg et al., 1997; Schwarz and Li, 1996; Kearsley et al., 1998). Gravity data from marine or terrestrial surveys, airborne gravity, satellite altimeter derived gravity and space gravity missions are widespread techniques that are able to supply large amounts of gravity data with unprecedented resolution and precision. However, this new data from satellite missions is bandwidth limited and marine gravity data or airborne gravity data could be used to recover the high frequencies of the gravity spectrum (Kern et al., 2003). Moreover, its usefulness will be limited in costal areas where altimeter data suffers for large errors that can contaminate the derived gravity anomalies (Deng et al., 2002; Rodriguez-Velasco et al., 2002). By other hand, marine gravity anomalies are irregularly distributed on sea, with sparse tracks crossing the sea, but with a clear densification in coastal areas and near continental margin. Clearly, both data sets are complementary in geographic data distribution and on geographic data quality, demanding we pursue methods to assimilate both into a better gravity data model that incorporates high frequency information and precision from marine gravity data with the high spatial coverage and (in some areas) less precise data from the satellite derived gravity anomalies. The great challenge of the integration of multi-source gravity

Copyright (c) The Society of Geomagnetism and Earth, Planetary and Space Sciences (SGEPSS); The Seismological Society of Japan; The Volcanological Society of Japan; The Geodetic Society of Japan; The Japanese Society for Planetary Sciences; TERRAPUB data is to derive a gravity model that incorporates the most accurate data with the best spatial resolution.

There are basically two main approaches for the combination of multi-source gravity data: one that operates on spatial domain working on the observation level and other that operates on the frequency domain combining data spectra. A solution for the combination of more than two data sources in the frequency domain was proposed by Kern et al. (2003). In this paper, a strategy is presented for the combination of heterogeneous data using spectral weights. This method relies on Least Squares spectral combination and requires the determination of spectral weights that are assumed to be error degree variances of local anomaly data and from satellite data. The drawback of this method is the required knowledge of the local data degree variances that are not known and must be estimated. Besides that, this method requires a regular distribution of observation data that it is not verified for shipborne surveys or even for airborne surveys.

On spatial domain, an operational strategy for combining different quality levels of gravity anomalies and different data resolution was already suggested by Strykowski and Forsberg (1998). The proposed method was an n-step process that maps less quality anomalies into better precision gravity anomalies data set, through the use of a residual surface computed from the difference between the first and the second model. In this method, a global precision is attributed for each data set discarding the possibility of local or regional variations in the precision given by different tracks surveyed in different epochs. Another similar, but more elaborated method, was proposed and analyzed by Olensen et al. (2002) in which satellite altimetric data 
and marine gravity data are mixed using least squares collocation. These authors present two different merging techniques, both based on collocation, that combines airborne gravity observations with either geoid observations derived from satellite altimetry or gravity derived from satellite altimetry. They verified that collocation approach, based on the combination of geoid satellite altimetry, gives slightly better results than the global mixing approach with only gravity data (satellite derived and airborne data). Although least squares collocation uses randomly distributed data, it requires intensive computation time and the proposed method requires the processing of satellite altimetric observations.

In this paper, we present an alternative method based on least squares Optimal Interpolation (Moritz, 1980; Boutier and Courtier, 2002) that combines two or more gravity data sets with their associated error variances into another gravity data set that is statistically optimal. This method is evaluated and its performance is compared with aforementioned methods (least squares collocation and drape technique). The study is carried out on the North-Atlantic Ocean between Iberian Peninsula and the Azores archipelago where satellite derived gravity anomalies from KMS02 (Andersen and Knudsen, 1998) and marine gravity data exists with different spectral and spatial resolutions, and involves different types of morphological structures from coastal areas, deep ocean to inter-islands areas. To access the accuracy of the resulting gravity model, airborne gravity data, not used in the merging process and recent shipborne gravity data was used. Also the geoid for this area was computed and its accuracy evaluated on sea by comparison with 10 year of Topex data and on land with GPS/levelling data.

\section{Mathematical Background}

Considering the real value of the gravity anomaly (not known) as $\Delta g^{r}(\phi, \lambda)$ and the background model as $\Delta g^{b}(\phi, \lambda)$, representing the apriori knowledge of the gravity anomaly field before the analysis is carried out, the analysis problem is to find a correction $\delta \Delta g(\phi, \lambda)$ that:

$$
\Delta g^{a}(\phi, \lambda)=\Delta g^{b}(\phi, \lambda)+\delta \Delta g(\phi, \lambda)
$$

is much closer to the real gravity anomaly field $\Delta g^{r}(\phi, \lambda)$ than the background model (Boutier and Courtier, 2002). The correction grid $\delta \Delta g(\phi, \lambda)$ is determined by the observation data. The observation data is irregularly distributed and is assembled into an observation vector with $\mathrm{N}$ data values. The best way to compare the observation data with the background model is through the use of a mapping function $f(\phi, \lambda)$ which calculates the model equivalent of the observation, and is called observation operator. In the general case, the observation vector can be a composite of gravity anomalies or any other gravity field related observation, like vertical deflections or sea surface height or geoid undulation. In this case, the observation operator will map the observation space into the background model using the fundamental equation of the physical geodesy (Heiskanen and Moritz, 1967) or any other related function. Assuming that errors are unbiased, the optimal least-squares estimation for the discrepancies is (Moritz, 1980):

$$
\Delta g^{a}=\Delta g^{b}+K\left(\Delta g^{\text {obs }}-f((\phi, \lambda))\right.
$$

and

$$
K=B F^{T}\left(F B F^{T}+D\right)^{-1}
$$

where $K$ is the weight matrix and the other error covariances are $B$ for the background model and $D$ for the observation, and $F$ is transformation matrix between the signals.

This is the solution for the global or regional analysis problem which requires inverting a matrix with a large dimension, equal to the number of observations $(\mathrm{N})$, which in earth sciences can be thousands or millions. The Optimal Interpolation (OI) analysis is a computational simplification of the matrix inversion in Eq. (3). In OI the weight matrix is simplified by assuming that only the closest observations determine the increment $\delta \Delta g(\phi, \lambda)$. OI implementation requires the knowledge of the background error covariance $B$ and the observation error covariance $D$. The matrix $D$ is supposing a diagonal matrix with the estimated observation variance. This estimated precision is the result of a marine (or terrestrial) gravity network adjustment or can be empirically based on the acquisition date, equipment and all other relevant information. The covariance $B$ portraits the correlation model that "correlates" the background model with the observation data. This matrix is defined locally for each local variable through an empirical autocorrelation function (Barzaghi and Sansò, 1983; Forsberg, 1984; Moritz, 1980) and must be positive definite.

This solution assumes that the discrepancies are unbiased, which means that the difference between the given set of observations and the background model is null (or close to) in the considered geographic domain. In gravity field modelling this hypothesis is very difficult to be verified. Shipborne gravity measurements are relative to some known base station and, therefore, any incorrect tie-in to that station may lead to a constant offset in all gravity data of that cruise. Assuming that background model is unbiased, the detected bias on the observations is removed and the above equations applied to the unbiased observations. It is important to detect and control the biases by analyzing previously the background model and the observations contrasting them with other source of data and/or related gravity field data. In this paper we have also analysed this key aspect by considering two different bias removal approaches.

\section{Gravity Data}

\subsection{Surveyed Gravity anomalies}

The original data bank, used in this study, was the result of a compilation from BGI (Bureau Gravimmetrique International, France) and NGDC (National Geophysical Data Centre, USA) data banks, and it covers an area with the following limits $\left(35^{\circ}<\varphi<45^{\circ}, 32^{\circ} \mathrm{W}<\lambda<6^{\circ} \mathrm{W}\right)$. Most of the data were acquired from USA, United Kingdom and France Institutions in the period from 1970 to 1990. This data bank was recently improved with a recent gravimetric campaign held in 1997 under the scope of the PDIC/C/Mar project (Fernandes et al., 1998) and AGMASCO project (Timmen et al., 2002). The complete data set, obtained from a simple merge of data files, was cleaned from repeated missions recorded in different data banks resulting in a data set with 197,702 data points and 776 tracks, with a 


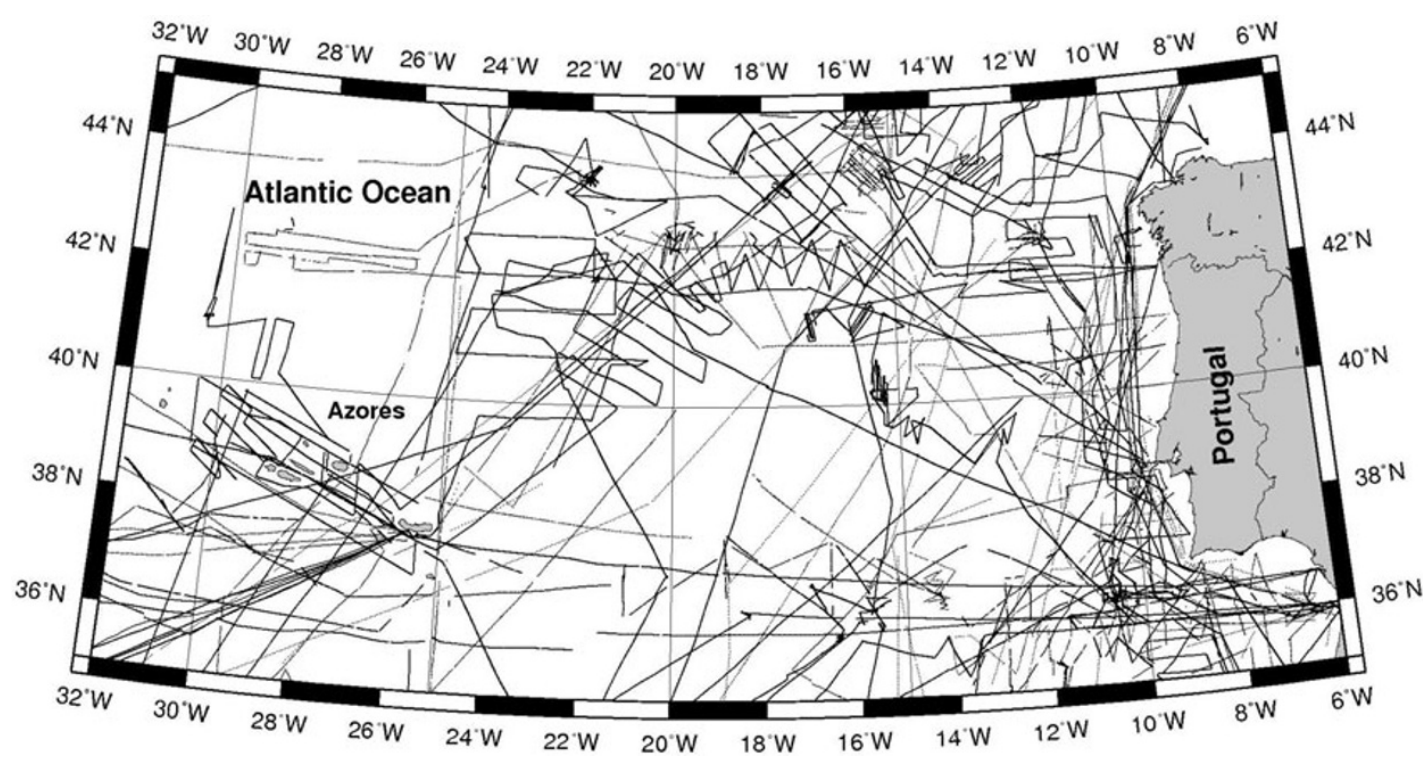

Fig. 1. Marine gravity data collected from BGI, NGDC and AGMASCO project in North-East Atlantic between Iberian Peninsula and Azores archipelago.

mean length of $199 \mathrm{~km}$ and a maximum length of $4264 \mathrm{~km}$. All data were transferred to IGSN71 system and the anomalies converted to GRS80. Geographical distribution of gravity data is depicted in Fig. 1. In our study area, marine gravity measurements obtained before the 80 's represents more than $66 \%$ of the total of available gravity observations.

Marine gravity data was validated and adjusted with specific software, written for this purpose, Catalao and Sevilla (2004). Spatial intersections between tracks (cross-over points) were determined (3992 intersections) and the external Crossover Errors (COE) were computed for each intersection. The external COE had a standard deviation of $10.81 \mathrm{mGal}$ and a minimum and maximum value of -94.87 $\mathrm{mGal}$ and $103.47 \mathrm{mGal}$, respectively. The amplitude of the COE is almost half of the gravity signal in this area, meaning that there are large discrepancies in the gravity observations belonging to different missions.

Track bias was determined through a global adjustment of the external COE and different weights to each observation equation were given. The minimum constraint adjustment was adopted with a one step global adjustment constraining to a zero bias the PDIC/C/MAR, surveyed in 1997. A standard deviation was attributed for each cruise or leg based on its surveying date in which for the most recent tracks (after 1980) a $2.5 \mathrm{mGal}$ standard deviation was considered, for the tracks between 1970 and 1980 a value of $7.5 \mathrm{mGal}$ was considered and for tracks before that date (1970) a value of $12.5 \mathrm{mGals}$ was considered for standard deviation.

After the adjustment, the standard deviation of the cross over errors reduces to $3.0 \mathrm{mGal}$, with a minimum and maximum reduced to -28.5 and $28.7 \mathrm{mGal}$, respectively. The adjustment solution (bias) was applied to each track and the associated individual (for each track) estimated standard deviation was assigned to each observation belonging to a track. The resulting file has 157,758 gravity anomaly observations with an associated standard deviation that does not reflect its own estimated precision but the overall quality of the track it belongs to.

\subsection{Satellite derived gravity anomalies}

There are several public domain altimetric derived gravity anomalies data sets with a global coverage. These models, in grid format, were computed directly from sea surface heights, KMS02 (Andersen et al., 1999), or through deflections of the vertical (Sandwell and Smith, 1997). KMS02 is the latest of a series of satellite derived gravity anomalies data sets supplied by Kort-og MatrikelStryrelsen and can be free downloaded from ftp.kms.dk. Numerous comparisons between marine observations and satellite altimetry derived gravity anomalies data sets have been presented (Andersen and Knudsen, 1998, Sandwell and Smith, 1997). The reported precision ranges from $3 \mathrm{mGal}$ to $14 \mathrm{mGal}$ as a function of the geographic location. In the North-Atlantic the reported precision for Sandwell and Smith (1997) model is $7.6 \mathrm{mGal}$ and for KMS model is $5.8 \mathrm{mGal}$ (Andersen and Knudsen, 1998). KMS02 shows the best agreement with ship-borne gravity data and because of this we have adopted this model (KMSO2) as our background model.

The model, KMS02, with a resolution of $0.033^{\circ}$, covers all marine study area overrunning land areas as Azores islands or some areas of Iberian Peninsula. Because of altimetric gravity data degradation on coastal regions, a mask with the coastal line with an offshore buffer of $20 \mathrm{~km}$ were constructed and used to clean all satellite data inside that area. Land data on Azores islands and Iberian Peninsula (see Table 1) was used to fulfil these blank areas and a final grid was constructed with satellite derived gravity anomalies over sea and observed anomalies over land areas, with the same correlation distance of $10 \mathrm{~km}$ used to compute KMS02 model. The resulting model has a resolution of $0.02^{\circ} \times 0.025^{\circ}$ corresponding to $2 \mathrm{~km}$, in agreement with the existing data resolution on land (Iberia and Azores). 
Table 1. Differences between KMS02 gravity and marine gravity data (in mGal).

\begin{tabular}{lcrrrr}
\hline & Number of points & Min & Max & Mean & Std. Dev. \\
\hline Land data & 43998 & -83.3 & 263.7 & 41.30 & 41.52 \\
KMS02 + land & 261627 & -182.9 & 359.4 & 19.90 & 39.70 \\
Marine data & 157428 & -171.1 & 357.5 & 19.26 & 44.10 \\
Difference & 157428 & -47.4 & 67.1 & 5.30 & 5.75 \\
\hline
\end{tabular}

\section{Merging by Optimal Interpolation}

The basic principle underlying OI algorithm is the existence of a background gravity model that completely covers all geographic domains. This model is the first guess of the gravity field and must be complemented with an associated error covariance model. This requirement is completely accomplished by the altimetric derived gravity anomalies, when complemented with land gravity data. In this circumstance, shipborne gravity data and its associated covariance error model are the observation set that will contribute to the improvement of the first guess model.

Accordingly with Eq. (2) the discrepancies between the background model and the observation set must be determined in the observation position. The background model is a discrete model, and the observation set is irregularly distributed, this means that they do not coincide, and an interpolation function must be derived to evaluate the background model in the observation position. Least squares prediction was used for this purpose with a correlation distance of $10 \mathrm{~km}$ and a covariance error model determined from the background gravity data. The background error covariance matrix is determined with a second a secondorder Markov covariance function of the form (Forsberg, 1984):

$$
C(\psi)=C_{0}\left(1+\psi / \phi_{1}\right) e^{-\psi / \phi_{1}}
$$

where $\psi$ is the spherical distance, $C_{0}$ is the background model variance and $\phi_{1}$ is the correlation distance determined from the background model. The discrepancies between the background model and the observations are listed in Table 1. In this Table is shown that both data sets have a similar behaviour with a similar amplitude and standard deviation and almost equal mean value. It is important to notice that in the background model is also included land gravity data that may reflect the difference in the standard deviation. Differences were computed only at marine gravity data points and exhibit a mean value of $5.3 \mathrm{mGal}$ and a standard deviation of $5.75 \mathrm{mGal}$ in agreement with the results presented for this area by Andersen and Knudsen (1998). Results of this comparison shows that there is no perfect agreement between observed gravity anomalies and altimeter derived ones, reaching differences of more than 60 $\mathrm{mGal}$. A residual mean value of $5.3 \mathrm{mGal}$ means that one of the data sets is biased and in the worst situation both are biased. Assuming that this residual is the result of shipborne datum inconsistencies and other bias-like effects, bias was removed by adding $-5.3 \mathrm{mGal}$ to all marine data, further referenced as OI-b. This approach could not be satisfactory once the gravity field is not homogeneous and local bias is detected. Alternative approach was achieved by fitting individually ship tracks to the KMS02 model by estimating bias and tilt to each track, further referenced as OI-t. After a careful editing process a crossover adjustment of the tracks was performed subject to the individual fitting to KMS02 model. Individual track discrepancies and a predefined threshold level was used to reject bad marine tracks.

The implementation of the optimal interpolation algorithm requires the definition of the background error covariances ( $B$ matrix) and the covariance error model for the observations ( $D$ matrix), see Eq. (3). $D$ matrix is a diagonal matrix with the observation covariance error estimated by the marine gravity adjustment. For each observation was assigned a covariance error value equal to the estimated precision of the track it belongs to. The background error covariance matrix is determined with a second-order Markov covariance function using Eq. (4). In Optimal Interpolation the weight matrix $K$ is simplified by assuming that only the closest observations determine the increment to apply to the background model. In this case, the selection of observations should afford all pertinent observations (with a significant weight) within a given radius around the background model point location. A mix approach based on an equal number of observations and a circular area restriction was adopted. This can be achieved by defining a minimum number of observations and introducing a localization delta function $\delta(\phi, \lambda)$ that acts as a switch in the error value determined between the background model and the observation data. Equation (2) assumes the following aspect:

$$
\Delta g^{a}=\Delta g^{b}+K\left(\left(\Delta g^{\text {obs }}-f(\phi, \lambda)\right) * \delta(\phi, \lambda)\right) .
$$

The localization function is a delta function that assumes zero or one as a function of the distance between observation point and background data location. In marine gravity surveys, observations are distributed along tracks resulting in an extreme anisotropic spatial distribution that must be taken into account in the adopted strategy for observations selection. Furthermore, the adopted covariance function is isotropic, and thus does not consider the anisotropic distribution induced gravity field anisotropies. Therefore, the selection process must be done in quadrants to guarantee a homogeneous distribution in azimuth with a minimum and maximum number of observations per quadrant. In this study case a minimum of 5 and a maximum of 20 observations per quadrant were selected. The turn off distance of this delta function should be chosen according to the residual field correlation distance and with the background model grid separation. The turn off distance was 6 arc min, which is the background model correlation distance and almost 6 times the background model grid separation. 


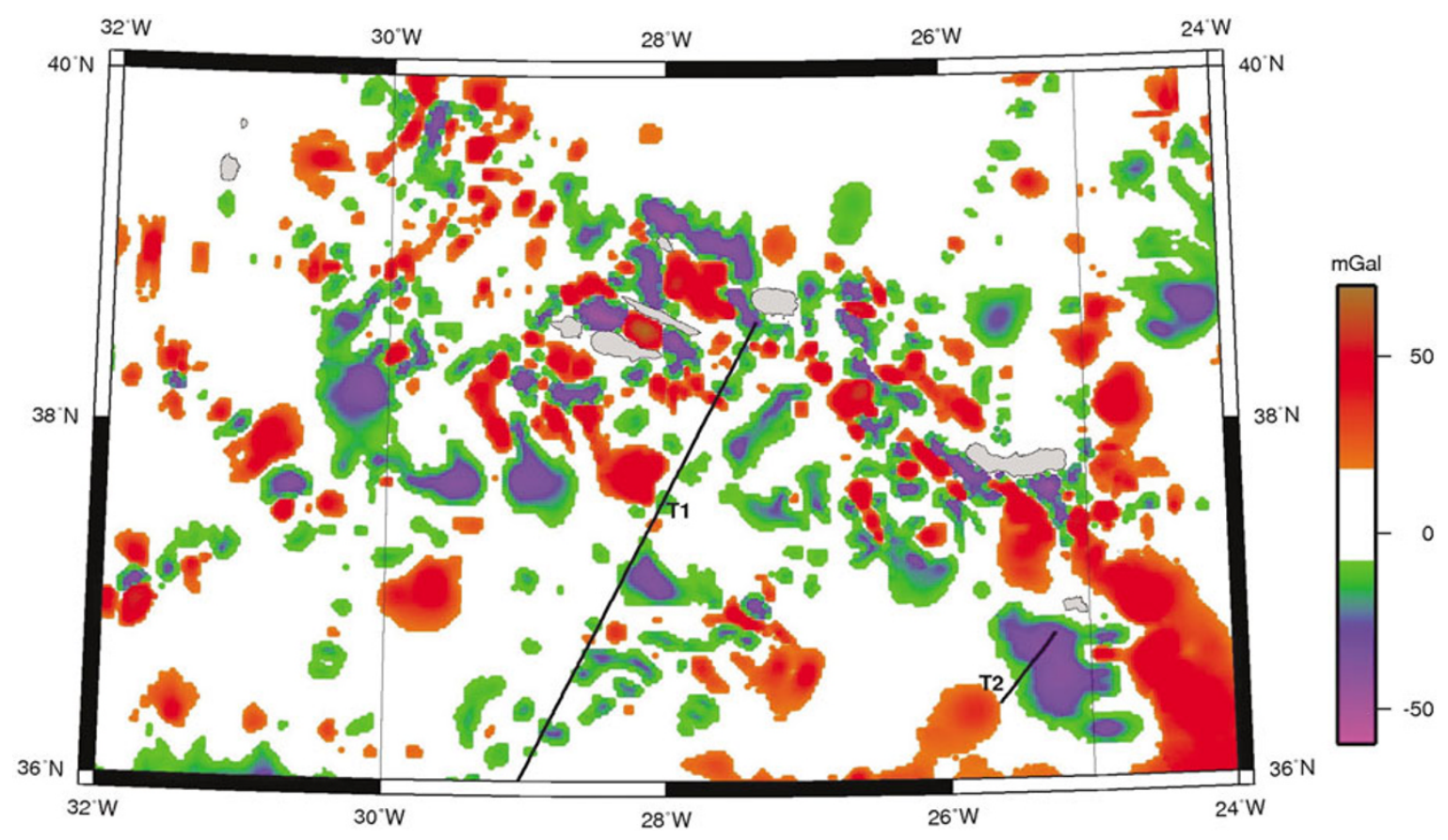

Fig. 2. Mapping function derived by OI applied to the background model. Two tracks (T1 and T2) are also plotted, used for evaluation purposes.

Table 2. Mapping function, original KMS02 gravity field, resulting Iberia-Azores Gravity Model (IAGRM), reduced to EGM96 model and residual gravity field after the removal of EGM96 and RTM effects (in mGal).

\begin{tabular}{lrrrc}
\hline & \multicolumn{1}{c}{ Min } & Max & Mean & Std. Dev. \\
\hline Mapping function & -56.8 & 68.6 & 0.20 & 3.97 \\
KMS02 & -181.9 & 359.7 & 18.18 & 38.62 \\
IAGRM & -178.8 & 340.6 & 18.39 & 38.74 \\
IAGRM-EGM96 & -118.4 & 209.0 & 0.09 & 18.58 \\
IAGRM-EGM96-RTM & -133.3 & 147.1 & 0.28 & 16.01 \\
\hline
\end{tabular}

Applying this methodology, we solved simultaneously for the extreme spatial irregular distribution of the marine data, for the continuity against neighbour background points and for far distance influence and inverse distance correlation problem. With a limited selected number observations, the weight matrix $K$ is also limited to the number of points (in this case a maximum of $4 \times 20=80$ ).

Appling the aforementioned methodology, and parameters, a correction surface was derived and mapped into the background model. This surface is presented in Fig. 2 limited to the Azores archipelago enhancing the most appealing aspects of the method. The mapping function has a minimum value of $-56 \mathrm{mGal}$ and a maximum value of $68 \mathrm{mGal}$ between S. Jorge and Pico islands. In this picture the white areas corresponds to areas where the mapping function is between $-5 \mathrm{mGal}$ and $5 \mathrm{mGal}$. The mapping function has extremely irregular behaviour acting on the low wavelength spectrum of the gravity field improving its spectral resolution. Statistics for this surface and for the final gravity field in the North-Atlantic are presented in Table 2, with a total of 541581 points. From the results of this table it is verified that there is no gain in the amplitude and the STD is almost the same when comparing KMS02 and IAGRM models. In fact, the background model (KMSO2) was only improved along the ship tracks within a few number of observations (about 13\%) and their impact is almost dissolved in the global data set. Even though, in a small area like the one depicted in Fig. 2, the statistic are also very similar but, analysing the differences between these two grids it was verified that the STD of the differences is $3.6 \mathrm{mGal}$. It is also verified that the residual gravity field, after the removal of long and short wavelengths (EGM96 model and RTM correction), is unbiased and the standard deviation was reduced to half of the original data. In picture 3 is depicted the final free air gravity anomaly model for the North Atlantic area between Iberia-Azores as a result of the data assimilation process.

\section{Analysis}

The precision and reproducibility of the final gravity model was evaluated by comparison with new marine gravity data surveyed after 1990, not included in the model, and with other gravity models derived by other methods (Drape technique and Least squares collocation). Besides, the effect of different merging methodologies is evaluated on geoid undulation, comparing with GPS/levelling sites on costal areas and with 10 years of TOPEX data.

The first evaluation was done by inspecting two different 


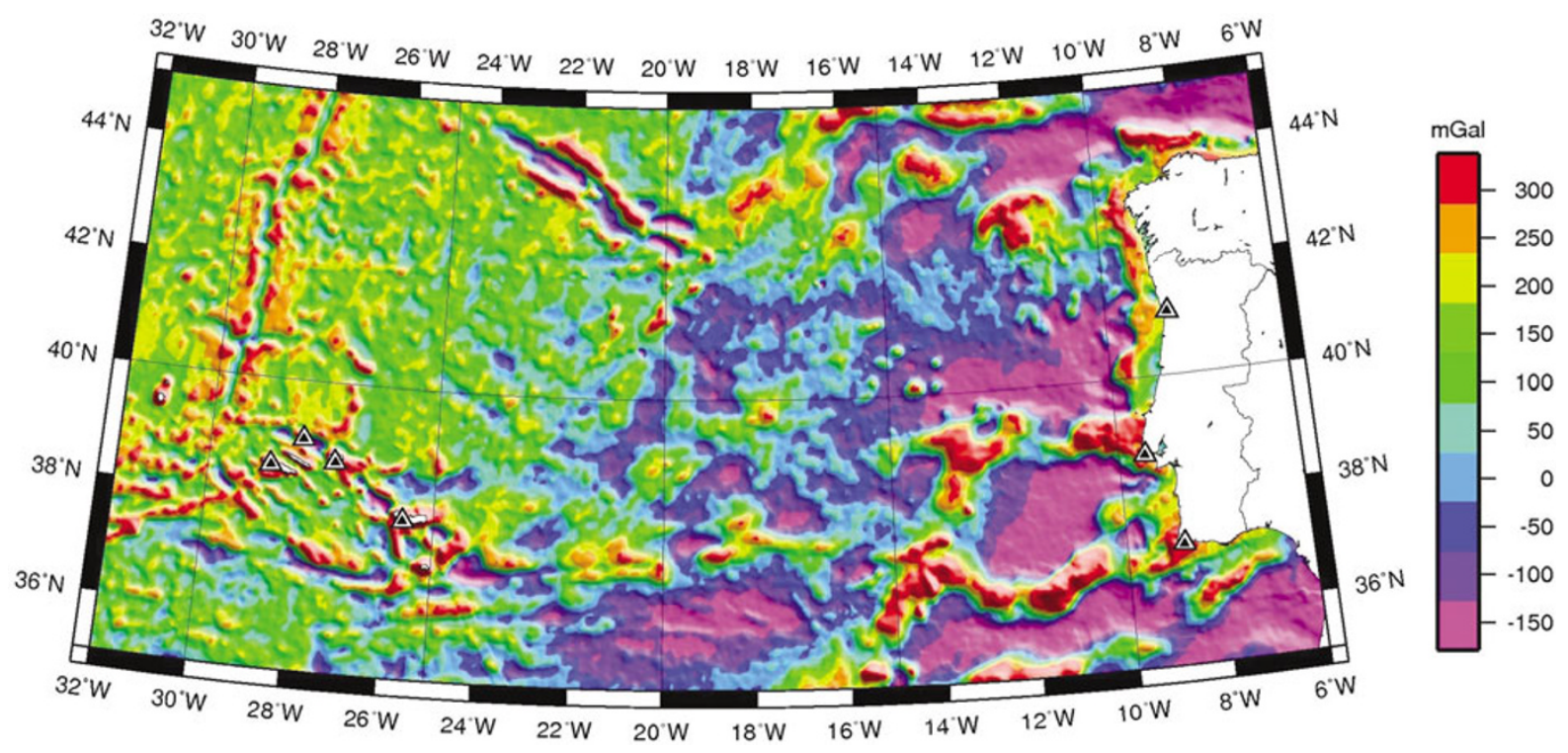

Fig. 3. Gravity field model in the North-Atlantic between Iberia and Azores archipelago resulting from the assimilation of marine gravity data into satellite derived gravity by optimal interpolation. GPS/levelling sites are presented as triangles.

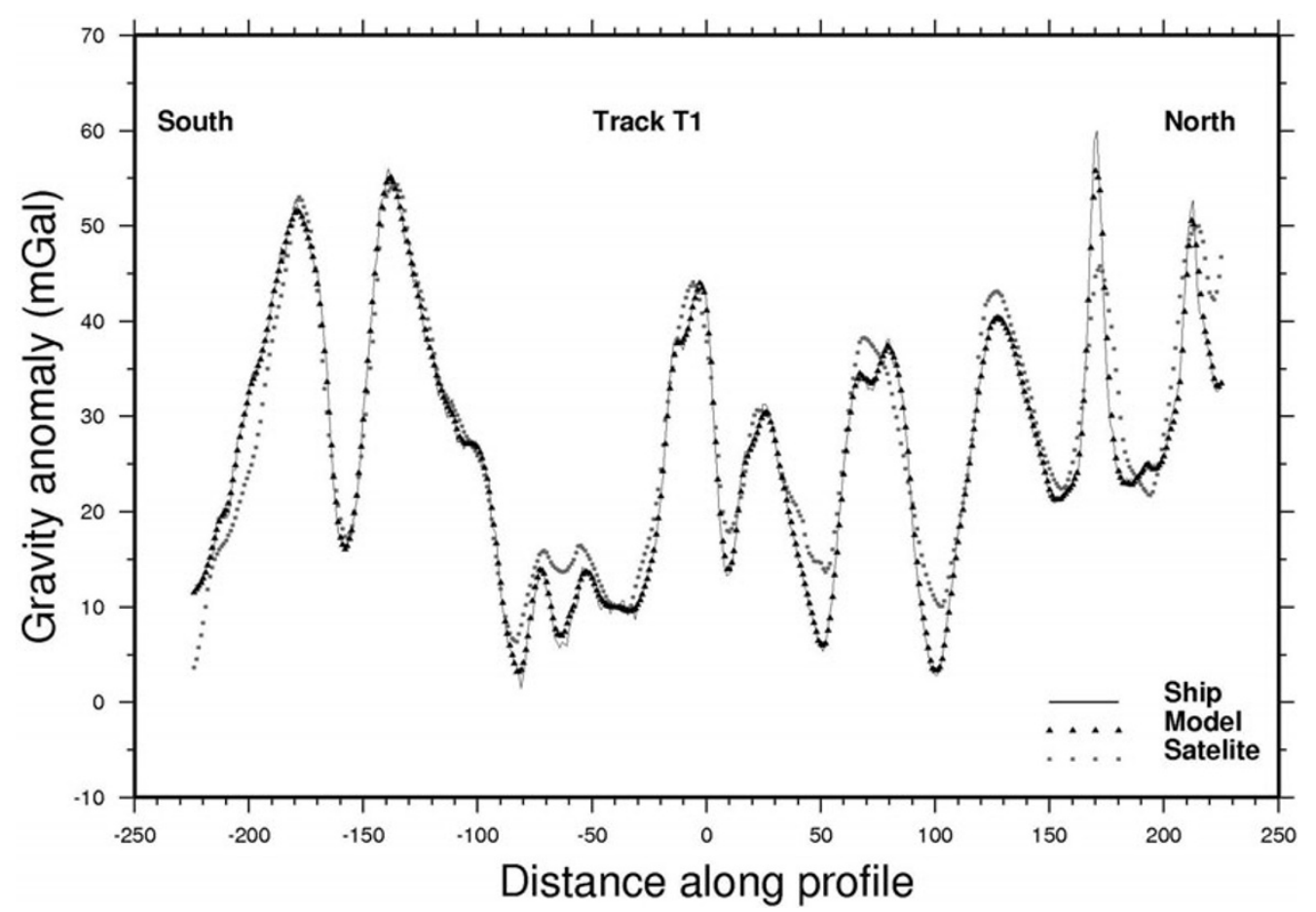

Fig. 4. Comparison of different gravity data types in a shipborne profile (T1) starting in Terceira island running to south-west.

tracks around Azores archipelago, with different estimated error variances. The first one is the most precise track, descending from north-east to south-west from Terceira Island (track T1, see Fig. 2) with an estimated precision of 1.5 $\mathrm{mGal}$. The second track is on the south of S. Maria Island and runs also from north to south with an error variance of $15.7 \mathrm{mGal}$ (track T2, see Fig. 2). Background, marine and final models were evaluated in both tracks and a set of profiles was drawn for track T1 and depicted in Fig. 4. In this figure is seen that there is an almost perfect agreement between satellite and marine gravity data confirming the superior quality of the background model (KMS02 satellite gravity) resolving accurately long wavelengths. In this case, due to the high precision of this marine track $(1.5 \mathrm{mGal})$ the derived gravity model assumes the shipborne data behaviour increasing signal amplitude and spectral resolution of the satellite data (background model).

In order to better visualize the short wavelength behaviour of the derived gravity model, in contrast with the satellite and shipborne data, two specific segments of track $\mathrm{T} 1$ and $\mathrm{T} 2$ were drawn in Fig. 5. Track T1 is more precise than the satellite data and in this case it was expected that the derived gravity model reflects this fact assimilating its behaviour. This is shown in Fig. 5(a) in which the 

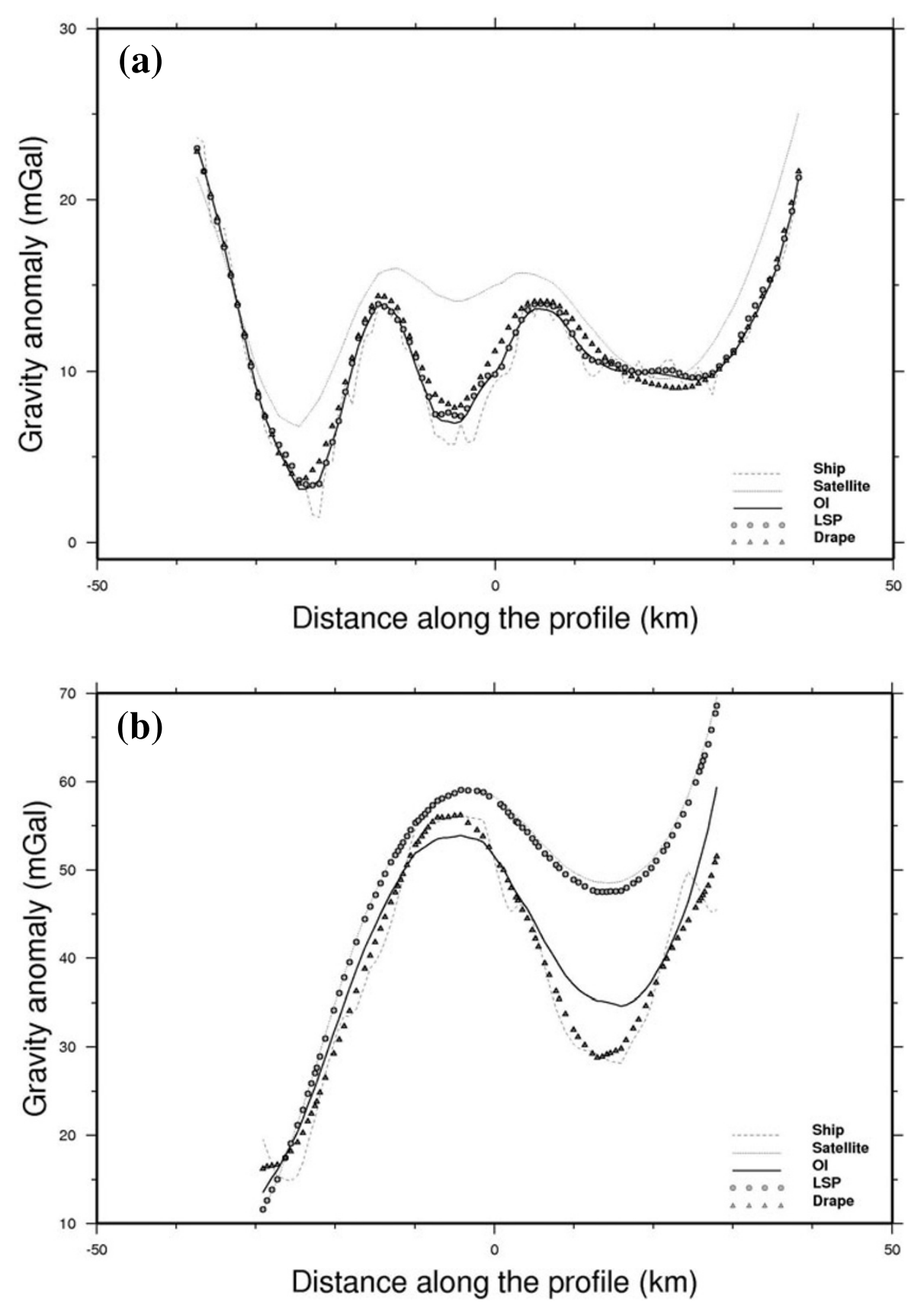

Fig. 5. (a) Short segment of most precise shipborne track (T1). (b) Short segment of less precise ship-borne track (T2). Shipborne data (Ship), background model (satellite), derived model (OI), least squares prediction model (LSP) and drape model (DRAPE) along these tracks are superimposed. Units $\mathrm{mGal}$ and $\mathrm{km}$.

final model sticks to the observation data. The opposite effect is seen for track T2, less precise than satellite data, in which the final model sticks to the background model, see Fig. 5(b). The final model tends to assume the behaviour of the most precise data as a compromise between the background and the observation error variance model. This effect was expected and highly desirable allowing the simultaneous combination of multi-source gravity data into a coherent gravity model, as long as the error variance are properly assign to each source.

The comparison was also done with other two methodologies: one step least squares prediction (Tscherning, 1985) and drape technique proposed by Strykowski and Forsberg (1998). These two methods are very similar to optimal interpolation scheme, differing in the formal implementation and in the error covariance models handling. In one step least squares prediction, the background model and the observation data are treated as an input signal with the same error covariance model defined for optimal interpolation. The error variance model is defined by Eq. (3), and the covariance values are $25 \mathrm{mGal}^{2}$ for the background data and a variable value assigned to each track by the crossover adjustment of the marine data. Drape technique was applied in a three step procedure: 1) determination of the residuals between background data and observation data; 2 ) softly grid the residuals; 3 ) drape these residuals into the observation data and merge with the background model. For comparison purposes we have used the same two tracks T1 
and T2. In Fig. 5 are also depicted the results of these two methodologies labelled as LSP (Least Squares Prediction) and DRAPE for the drape technique.

In one step least squares prediction both data sets (background model and observations) are treated at the same level and the resulting model is the best least squares estimates according to the assumed error variance model. This is particularly evident in the two selected tracks. In T1 track the observation data is much more precise than the background model and as a consequence the final model follows the observation data. In T2 track the background model (satellite data) is more precise than the observation data and the final model follows the background model (the satellite data). We have also analyzed a cross track profile and verified that the observation influence is limited to the along track direction with a rapid decay of the observation value. This is due to the disproportional amount of data used in the LSP process. An isolated track is surrounded by a large amount of data from the background model that envisages the estimation process even if a large precision is assigned to the observation data, limiting its influence to the along track direction.

In drape technique, the background error variance is not explicitly considered and the resulting model exhibits a better agreement with the observation data, irrespectively its quality. For the most precise track (T1) this corresponds to the expected behaviour of the final model, but for track T2 it is observed that the model follows the observation in a situation that the balance should be to the background model. The drape technique, assumes that the background model is of better quality than the observation data and it does not consider local variations on data quality. The proposed optimal interpolation scheme reveals a better agreement with the reality than the other two methodologies with a better balance between the weight of the background model and observation data. This is particularly true for irregular and sparse observations as it stands for marine gravity observations surveyed along tracks. For very dense marine gravity data coverage it is believed that these three methods will converge to a very similar solution, but in typical irregular marine data coverage with sparse tracks with known error variance, the optimal interpolation fine-tuned with the local data better reflects the reality.

The reproducibility of the final model was also analyzed numerically computing the misfit between these three different final models and the shipborne data. We have considered two situations: residuals of observations with standard deviation less than $5 \mathrm{mGal}$ (observations more precise than the background model) and residuals of observations with standard deviation greater than $5 \mathrm{mGal}$. In the first case, the three methods return a similar result, ranging the misfit from 1.9 to $2.1 \mathrm{mGal}$, on the standard deviation, which may be considered as an internal error evaluation. The major difference between these methods relies on the assimilation of less precise observations (with $\sigma>5 \mathrm{mGal}$ ) into the final model. Drape method, with the smaller standard deviation (2.9 mGal), almost follows the observation data, even in this case where the background model is more precise than the observation data. An opposite behaviour is verified with LSP where a value of $6.9 \mathrm{mGal}$, on the standard deviation, reveals that ship borne data (observations) were not considered in the assimilation process. The OI algorithm (with $4.8 \mathrm{mGal}$ ) reveals a better balanced result with a compromise between the standard deviation of the observation data and the background model. These numerical results and the previous graphical representation, of the three methods, demonstrates the skill of the OI method for dealing with multi-source gravity data with different stochastic models assimilating optimally into a coherent gravity data model.

External error assessment was evaluated by comparing with recent marine surveys obtained from the new release of NGDC geophysical data, version 4.1.18. In this recent release, 5 new marine surveys after 1990, that were not included in the model, were identified (2 in Azores region and 3 close to Iberia). Also, an airborne survey was done in Azores archipelago (Olesen and Forsberg, 1999) in the aim of AGMASCO project, and was used for error evaluation. In this campaign Olesen and Forsberg (1999) reported several problems related with a detected bias between airborne and shipborne gravity data which motivate the exclusion of this data in our merging process. Some lines showed a bias between 10 to $15 \mathrm{mGal}$ when compared to shipborne gravimetry. Nevertheless, we have considered this data as an external evaluator. Results of these comparisons are presented in Table 3. In this table is seen that the standard deviation of the error is almost always smaller for merged models than for the original KMS02 model, with the LSP and OI-t performing better than drape and OI-b methods. There are two situations where it seems there is no improvement (19100041 and 19180010), using OI-t and LSP, which corresponds to areas where there were no marine data. In this case, OI-b and Drape methods corrupt the original model. Both methods suffers from a bias problem that are not properly handle and are passed through the original model introducing discontinuities in the final gravity model, along the biased track. The improvement is only obtained in areas where marine data is available but is also a function of the method used and also the quality of this new marine gravity data. It is seen that OI-t and LSP approaches have the ability to maximize the observation usefulness by propagating its information in space even for randomly distributed data. Survey 67010202 runs through Azores archipelago crossing the inter-island area where altimeter data suffers for several gross errors. Along this track, the standard deviation reduces from $12 \mathrm{mGal}$ for KMS02 model to $2.2 \mathrm{mGal}$ and $2.8 \mathrm{mGal}$ revealing an extreme improvement case. The small standard deviation obtained for all methods indicates that the area covered by this track was already improved by other (or others) marine tracks. In general, we are able to say that in costal areas where satellite derived gravity data is less accurate, the inclusion of shipborne gravity data and/or airborne gravity data using OI-t approach will improve the final gravity model to an accuracy of about $3 \mathrm{mGal}$.

As it was expected, airborne data shows a greater disagreement than shipborne data in Azores area, but even so, it is verified that the fit of airborne data is better for all merging methods than for KMS02 model. Nonetheless, airborne data is not enough accurate to be included in the final model.

The effect of different merging methodologies on geoid was also evaluated. Four gravimetric geoid solutions were 
Table 3. Standard deviation of the comparison between derived models and new surveys performed after 1990 and also with airborne data from Agmasco project (in mGal). Survey name is the survey code in NGDC data set and $\mathrm{N}$ the number of observations.

\begin{tabular}{lccccccc}
\hline & Surv. Name & $\mathrm{N}$ & Kms02 & OI-b & Drape & LSP & OI-t \\
\hline \multirow{2}{*}{ Azores } & 67010208 & 3865 & 4.7 & 3.2 & 3.3 & 3.3 & 3.1 \\
& 67010202 & 2083 & 12.0 & 2.2 & 2.4 & 2.3 & 2.8 \\
\multirow{3}{*}{ Iberia } & 19100041 & 1446 & 5.9 & 6.4 & 6.7 & 5.9 & 6.2 \\
& 67010183 & 6456 & 7.9 & 2.7 & 2.9 & 2.9 & 3.2 \\
& 19180010 & 3391 & 3.8 & 4.9 & 5.1 & 3.6 & 3.8 \\
Airborne & Agmasco & 10005 & 7.5 & 6.9 & 7.2 & 6.7 & 6.8 \\
\hline
\end{tabular}

determined using different gravity models derived from abovementioned methodologies. Classical remove-restore methodology was used and the Stokes integral formula was applied. EGM96 geopotential model (Lemoine et al., 1997) to degree and order 360 was used as a reference model and short wavelength topographic effects were computed from the RTM (Residual Terrain Model) (Forsberg and Tscherning, 1981) using a medium precision digital terrain model with $500 \mathrm{~m}$ spatial resolution spanning all area. To evaluate the resulting geoid, the absolute geoid undulations were compared with those obtained by 7 GPS/levelling sites on Iberian coast and on Azores islands, see Fig. 3 for site location. The three Iberian GPS stations are GAIA, CASC and LAGO permanent EUREF stations nearby tide gauges. The other 4 GPS stations, in Azores, TERC, FAIM, GRAC and PDEL (IGS station), are also permanent stations with coordinate repeatability of 2-6 $\mathrm{mm}$ for horizontal and vertical components (Navarro et al., 2003). On sea, geoid was compared with sea surface heights obtained from 10 years of TOPEX data (Table 4). The comparison determined by GPS/levelling shows that there are significant differences between the four solutions in coastal areas with a maximum of $4 \mathrm{~cm}$ between drape technique and OI-t. There is also a significant difference of $3 \mathrm{~cm}$ between OI-b and OI-t. These two datasets differ only on the used method for bias removal, revealing that better results are obtained when bias and tilt are removed through individual marine track fitting to the background model. The same conclusion can be drawn from results on sea. In this case, the results obtained from OI-t are almost 2 times better than those obtained with drape technique and $1 \mathrm{~cm}$ when compared with OI-b.

Difference between LSP and OI is more practical than conceptual. In fact, both have the same mathematical formulation and stochastic model, the only difference resides on the way data is handled. In LSP, background data and new data are treated at the same level (although with different error models) and the system has to manage a large number of data (even millions). In OI approach, new data is treated has a correction to the background model and the system has only to manage a reduced number of observations (the new data). The results of both approaches should be similar as it was verified.

\section{Conclusions}

In this study, marine gravity data and satellite derived gravity data from KMSO2 were combined to derive a re-
Table 4. Comparison of different geoid solutions computed from different merging approaches with 10 year of Topex data (Marine) and GPS/levelling (Land). Presented results are the residual standard deviation in $\mathrm{cm}$.

\begin{tabular}{lcccc}
\hline & OI-b & Drape & LSP & OI-t \\
\hline Marine & 9.5 & 15.8 & 7.7 & 8.2 \\
Land (coast) & 5.0 & 6.3 & 2.1 & 2.1 \\
\hline
\end{tabular}

liable gravity model that will be useful both for geophysical studies and geoid determination. It was shown that combined gravity data improves the precision of the derived geoid undulation (both on land and on sea) and the fit to precise shipborne gravity data. This result was particularly obvious in coastal and inter-island areas where satellite derived gravity anomalies may contain errors.

It was verified that assimilation with only a regional bias removal (OI-b), introduces discontinuities on the background model delimited by the track envelop (defined by the track influence area). It is likely to be a bias problem that was successfully solved in the OI-t approach where individual tracks are fitted to the background model by a bias and tilt parameter. A similar problem exists in drape technique where the method assumes that the new data is of better quality that the background model which is known not to be true and depends on the satellite derive gravity and on the marine data quality. It was also clear that OI-t and LSP have the same behaviour with a similar performance. Using these methods, the improvement of the final gravity model when compared to the original KMS02 model, is dependent on the geographic area but not on the marine data quality used in the model. It means that there is no model degradation and the improvement can reach $10 \mathrm{mGal}$ in rms at inter-island areas.

The influence of different merging approaches on the geoid determination was evaluated and it was verified that there exist a considerable influence of the method on the geoid precision. Drape and OI-b approaches are slightly worst than LSP and OI-t. It seems that a high frequency noise was introduced on the model, by means of the inclusion of biased gravity data, which are likely to propagate right through the geoid causing its degradation. With OI-t or LSP, geoid solution was improved in this area more than $7 \mathrm{~cm}$ on sea and $4 \mathrm{~cm}$ on land when compared to other solutions. This results demonstrates the skill of OI-t approach for dealing with multi-source gravity data, assimilating op- 
timally into a coherent gravity data model with a considerable recover of the high frequency spectrum of the gravity field with a low computational coast when compared with least squares prediction.

Acknowledgments. We are grateful to Eng. Helena Koll from Instituto Geográfico Português for supplying land gravity data on Portugal mainland and we also thank to Dra. Luísa Bastos for the airborne gravity data. The figures have been produced using the GMT software (Wessel and Smith, 1991). This work was funded by FCT (Portuguese Foundation for Sciences and Technology) through KARMA project (POCTI/CTE-GIN/57530/2004).

\section{References}

Andersen, O. B. and P. Knudsen, Global marine gravity field from ERS1 and Geosat geodetic mission altimetry, J. Geophys. Res., 103(C4), 8129-8137, 1998.

Andersen, O. B., P. Knudsen, S. Kenyon, and R. Trimmer, Recent improvement in the KMS global marine gravity field, Bollettino Geofisica Teorica ed Applicata, 40, 369-377, 1999.

Barzaghi, R. and F. Sansò, Sulla stima empirica della funzione di covarianza, Bollettino di Geodesia e Scienze Affini, XLII(4), 389-415, 1983.

Boutier, F. and P. Courtier, Data assimilation concepts and methods, Meteorological Training Course Lecture Series, ECMWF, 2002.

Catalao, J. and M. J. Sevilla, Inner and minimum constraint adjustment of marine gravity data, Computer and Geosciences, 30(9-10), 949-957, DOI: 10.1016/j.cageo.2004.06.004, 2004.

Childers, V. A., D. C. McAdoo, J. M. Brozena, and S. L. Laxon, New gravity data in the artic Ocean: Comparison of airborne and ERS gravity, J. Geophys. Res., 106, 8871-8886, 2001.

Deng, X., W. E. Featherstone, C. Hwang, and P. A. M. Berry, Estimation of contamination of ERS-2 and Poseidon satellite radar altimetry close to the coasts of Australia, Marine Geodesy, 25, 249-271, DOI: 10.1080/01490410290051572, 2002.

Fernandes, M. J., A. Gidskehaug, D. Solheim, M. Mork, P. Jaccard, and J. Catalao, Gravimetric and Hydrographic campaign in Azores, in Proceedings of the I Luso-Spanish Assembly in Geodesy and Geophysics, Almeria, Spain, 9-13 Feb., University of Almeria, p. 113, 1998.

Forsberg, R., Local covariance functions and density distributions, Dept. of Geodetic Science and Surveying, Rep. No. 356, The Ohio State University, Columbus, Ohio, 1984.

Forsberg, R. and J. M. Brozena, The Greenland airborne gravity projectcomparison of airborne and terrestrial gravity data, in BGI, Bulletin D'Information No. 71, Workshop on marine gravity data validation, Toulouse, Oct. 27-28, 55-58, 1992.

Forsberg, R. and C. C. Tscherning, The use of height data in gravity field approximation by collocation, J. Geophys. Res., 86(B9), Sept. 10, 78437854, 1981.

Forsberg, R., K. Hehl, L. Bastos, A. Giskehaug, and U. Meyer, Development of an airborne geoid mapping system for coastal oceanography (AGMASCO), in Proceedings of the International Symposium on Gravity, Geoid and Marine Geodesy, GRAGEOMAR, edited by J. Segawa, H. Fujimoto, and S. Okubo, The University of Tokyo, Tokyo, Sept. 30-Oct.
5, 1996, Springer-Verlag, pp. 163-170, 1997.

Heiskanen, W. A. and H. Moritz, Physical Geodesy, W. H. Freeman and Company, San Francisco, 1967.

Kearsley, A. H. W., R. Forsberg, A. Olesen, L. Bastos, K. Hehl, U. Meyer, and A. Gidskehaug, Airborne gravimetry used in precise geoid computations by ring integration, Journal of Geodesy, 72, 600-605, 1998.

Kern, M., P. Schwarz, and N. Sneeuw, A study on the combination of satellite, airborne, and terrestrial gravity data, Journal of Geodesy, 77, 217-225, DOI 10.1007/s00190-003-0313-x, 2003.

Lemoine, F. G., D. E. Smith, L. Kunz, R. Smith, E. C. Pavlis, N. K. Pavlis, S. M. Klosko, D. S. Chinn, M. H. Torrence, R. G. Williamson, C. M. Cox, K. E. Rachlin, Y. M. Wang, S. C. Kenyon, R. Salman, R. Trimmer, R. H. Rapp, and R. S. Nerem, The development of the NASA GSFC and NIMA Joint Geopotential Model, in Proceedings of the International Symposium on Gravity, Geoid and Marine Geodesy, GRAGEOMAR, edited by J. Segawa, H. Fujimoto, and S. Okubo, The University of Tokyo, Tokyo, Sept. 30-Oct. 5, Springer-Verlag, pp. 461-469, 1997.

Moritz, H., Advanced Physical Geodesy, 500 pp., H. Wichmann Verlag, Karlsruhe, 1980.

Navarro, A., J. Catalao, J. M. Miranda, and R. M. S. Fernandes, Estimation of the Terceira island (Azores) main strain rates from GPS data, Earth Planets Space, 55(10), 637-642, 2003.

Olesen, A. V. and R. Forsberg, Azores airborne gravity processing, Personal communication, Copenhagen, March 1999.

Olesen, A. V., O. B. Andersen, and C. C. Tscherning, Merging airborne gravity and gravity derived from satellite altimetry: test cases along the coast of Greenland, Studia Geophys. Geod., 46, 387-394, 2002.

Rodriguez-Velasco, G., M. J. Sevilla, and C. Toro, Dependence of mean sea surface from altimeter data on the reference model used, Marine Geodesy, 25, 289-312, DOI:10.1080/01490410290051590, 2002.

Sandwell, D. and W. H. F. Smith, Marine gravity anomaly from Geosat and ERS1 satellite altimetry, J. Geophys. Res., 102(B5), 10039-10054, 1997.

Schwarz, K. P. and Y. Li, What can airborne gravity contribute to geoid determination?, J. Geophys. Res., 101(B8), 17873-17881, 1996.

Strykowski, G. and R. Forsberg, Operational Merging of Satellite, Airborne and Surface Gravity Data by Draping Techniques, in Geodesy on the Move-gravity, geoid, geodynamics and Antarctica, Proceedings IAG scientific assembly, Rio de Janeiro, Sept 3-9 1997, Forsberg, Feissel and Dietrich (eds.), IAG symposia 119, pp. 243-248, Springer Verlag, Berlin, 1998.

Timmen, L., L. Bastos, R. Forsberg, A. Gidskehaug, and U. Meyer, Airborne Gravity Field Surveying for Oceanography, Geology and Geodesy-Experiences from AGMASCO, in IAG Symposia, Volume 121, Springer Verlag, 2002.

Tscherning, C. C., Local approximation of the gravity potential by least squares collocation, in Proceedings of the International Summer School on Local Gravity Field Approximation, edited by K. P. Schwarz, Beijing, China, Aug. 21-Sept. 4, 1984, Pub. 60003, Univ. of Calgary, Calgary, Canada, pp. 277-362, 1985.

Wessel, P. and W. Smith, Free software helps map and display data, Eos Trans. AGU, 72, 441, 1991.

J. Catalao (e-mail: jcfernandes@fc.ul.pt) 